\title{
Existence Analysis of Traveling Wave Solutions for a Generalization of KdV Equation
}

\author{
Yao Long and Can Chen \\ College of Mathematics, Honghe University, Mengzi, Yunnan 661100, China \\ Correspondence should be addressed to Yao Long; longyaoteacher@yahoo.com.cn
}

Received 12 September 2012; Accepted 16 November 2012

Academic Editor: Salvatore Alfonzetti

Copyright (C) 2013 Y. Long and C. Chen. This is an open access article distributed under the Creative Commons Attribution License, which permits unrestricted use, distribution, and reproduction in any medium, provided the original work is properly cited.

\begin{abstract}
By using the bifurcation theory of dynamic system, a generalization of KdV equation was studied. According to the analysis of the phase portraits, the existence of solitary wave, cusp wave, periodic wave, periodic cusp wave, and compactons were discussed. In some parametric conditions, exact traveling wave solutions of this generalization of the KdV equation, which are different from those exact solutions in existing references, were given.
\end{abstract}

\section{Introduction}

In 1995, Fokas [1] derived a generalization of KdV equation

$$
u_{t}+u_{x}+v u_{x x t}+\beta u_{x x x}+\alpha u u_{x}+\frac{1}{3} \alpha v\left(u u_{x x x}+2 u_{x} u_{x x}\right)=0
$$

which from physical considerations via a methodology introduced by Fuchssteiner and Fokas [2]. Equation (1) can also be deriven by the approaches described in [3]. By using the bifurcation theory of dynamic system (see [4-7] and references cited therein), it is easy to investigate exact traveling wave solutions of (1) under different kinds of parametric conditions. For example, in [8], by using the bifurcation theory of dynamic system, some subsection-function and implicit function solutions such as compactons, solitary waves, smooth periodic waves, and nonsmooth periodic waves with peaks as well as the existence conditions have been presented by $\mathrm{Bi}$. By using the same method, Li and Zhang [9] studied a generalization form of the modified $\mathrm{KdV}$ equation, which is more complex than (1). In [9], the existence of solitary wave, kink and antikink wave solutions, and uncountably infinitely many smooth and nonsmooth periodic wave solutions is discussed. By using the improved method named integral bifurcation method [10, 11], Rui et al. [12] obtain all kinds of soliton-like or kink-like wave solutions, periodic wave solutions with loop or without loop, smooth compacton-like periodic wave solution, and nonsmooth periodic cusp wave solution for (1). The integral bifurcation method possessed some advantages of the bifurcation theory of the planar dynamic system $[6,13]$ and auxiliary equation method (see $[14,15]$ and references cited therein), it is easily combined with computer method [11] and useful for many nonlinear partial differential equations (PDEs) including some PDEs with high-power terms, such as $K(m, n)$ equation [16]. However, we cannot discuss the existence of traveling wave solutions by using this method. Therefore, the bifurcation theory of the planar dynamic system bulks large with the existence analysis of traveling wave solutions of nonlinear PDEs.

In this paper, by using the bifurcation theory of dynamic system and a translation transformation, we will restudy (1). According to the analysis of the phase portraits, the existence of solitary wave, cusp wave, periodic wave, periodic cusp wave, and compactons will be discussed. The obtained results are different from those in $[8,12]$.

\section{Bifurcations of Phase Portraits of the System and the Existence of Traveling Wave Solutions for (1)}

2.1. Bifurcations of Phase Portraits for the 2-Dimensional Planar System of (1). In this subsection, we discuss the first integral and bifurcations of phase portraits of (1). 
Making a traveling wave transformation $u=\phi(\xi)+\lambda$ with $\xi=x-c t$, (1) can be reduced to the following ordinary differential equation:

$$
\begin{gathered}
(1-c) \phi^{\prime}+(\beta-c v) \phi^{\prime \prime \prime}+\alpha(\phi+\lambda) \phi^{\prime} \\
+\frac{1}{3} \alpha v\left[(\phi+\lambda) \phi^{\prime \prime \prime}+2 \phi^{\prime} \phi^{\prime \prime}\right]=0 .
\end{gathered}
$$

We call the solution $\phi(\xi)+\lambda$ traveling wave solution.

Integrating (2) with respect to $\xi$, we have

$$
\begin{aligned}
& \left(\beta-c v+\frac{1}{3} \alpha v \lambda+\frac{1}{3} \alpha v \phi\right) \phi^{\prime \prime} \\
& \quad=g+(c-1-\alpha \lambda) \phi-\frac{1}{2} \alpha \phi^{2}-\frac{1}{6} \alpha v\left(\phi^{\prime}\right)^{2},
\end{aligned}
$$

where $g$ is an integral constant. Let $d \phi / d \xi=y$. Thus, (3) can be rewritten as the following two-dimensional planar system:

$$
\begin{gathered}
\frac{d \phi}{d \xi}=y \\
\frac{d y}{d \xi}=\frac{g+(c-1-\alpha \lambda) \phi-(1 / 2) \alpha \phi^{2}-(1 / 6) \alpha v y^{2}}{\beta-c v+(1 / 3) \alpha v \lambda+(1 / 3) \alpha v \phi}
\end{gathered}
$$

which is a singular system. Equation (4) has the following first integral:

$$
\begin{aligned}
H(\phi, y) \equiv & (3 c \nu-3 \beta-\alpha v \lambda-\alpha v \phi) y^{2} \\
& -\alpha \phi^{3}+3(c-1-\alpha \lambda) \phi^{2}+6 g \phi=h .
\end{aligned}
$$

Because the $d y / d \xi$ is not defined when $\beta-c \nu+(1 / 3) \alpha v \lambda+$ $(1 / 3) \alpha v \phi=0$, so we make a transformation as follows:

$$
d \xi=\left(\beta-c \nu+\frac{1}{3} \alpha \nu \lambda+\frac{1}{3} \alpha \nu \phi\right) d \tau,
$$

where $\tau$ is a parameter. Under the transformation (6), (4) becomes the following regular system:

$$
\begin{gathered}
\frac{d \phi}{d \tau}=\left(\beta-c v+\frac{1}{3} \alpha v \lambda+\frac{1}{3} \alpha v \phi\right) y, \\
\frac{d y}{d \tau}=g+(c-1-\alpha \lambda) \phi-\frac{1}{2} \alpha \phi^{2}-\frac{1}{6} \alpha v y^{2} .
\end{gathered}
$$

Obviously, (7) has the same first integral as (4) which is (5).

Obviously, system (7) has two equilibrium points $M_{1,2}\left(\phi_{1,2}, 0\right)$ on $\phi$-axes, where $\phi_{1,2}=((c-1-\alpha \lambda) \pm$ $\sqrt{\left.(c-1-\alpha \lambda)^{2}+2 \alpha g\right)} / \alpha$. System (7) has two equilibrium points $S_{ \pm}\left(\phi_{s}, Y_{1,2}\right)$ on singular line $\phi=$ $\phi_{s}$, where $\phi_{s}=(3 c v-3 \beta-\alpha v \lambda) / \alpha v, Y_{1,2}=$ $\pm \sqrt{3 \alpha v\left(2 g+2(c-1-\alpha \lambda) \phi_{s}-\alpha \phi_{s}^{2}\right)} / \alpha v$. By using (5), we obtain Hamiltonian of these equilibrium points as follows:

$$
\begin{aligned}
& h_{1,2} \equiv H\left(\phi_{1,2}, 0\right)=-\alpha \phi_{1,2}^{3}+3(c-1-\alpha \lambda) \phi_{1,2}^{2}+6 g \phi_{1,2}, \\
& h_{3} \equiv h_{s}=H\left(\phi_{s}, Y_{1,2}\right)=\left(3 c v-3 \beta-\alpha v \lambda-\alpha v \phi_{s}\right) Y_{1,2}^{2}-\alpha \phi_{s}^{3} \\
&+3(c-1-\alpha \lambda) \phi_{s}^{2}+6 g \phi_{s} .
\end{aligned}
$$

According to the characteristic and the relation of Hamiltonian of each equilibrium points, we obtain three bifurcations of parameters as follows:

$$
\begin{gathered}
g_{1}=-\frac{c(c-2-2 \alpha \lambda)+(1+\alpha \lambda)^{2}}{2 \alpha}, \\
g_{2}=\frac{(3 c v-3 \beta-\alpha v \lambda)(2 v+c v-3 \beta+\alpha v \lambda)}{2 \alpha v^{2}}, \\
g_{3}=\frac{(3 v+2 \alpha v \lambda-3 \beta)(4 c v-v-2 \alpha v \lambda-3 \beta)}{8 \alpha v^{2}} .
\end{gathered}
$$

Under different parametric conditions, that is to say, according to different bifurcations of parameters, in the different area of parameters and on the different bifurcation curves, we derive all kinds of bifurcations of phase portraits for the system (7), which are shown in Figures 1 and 2.

Suppose that $u(x, t)=\phi(\xi)+\lambda$ is a continuous traveling wave solution of (1) for $\xi \in(-\infty, \infty)$, and $\lim _{\xi \rightarrow \infty} \phi(\xi)=$ $a, \lim _{\xi \rightarrow-\infty} \phi(\xi)=b$. It is well known that $(\mathrm{i}) \phi(x, t)$ is called a solitary wave solution if $a=b$; (ii) $\phi(x, t)$ is called a kink or antikink solution if $a \neq b$. Usually, a solitary wave solution of a nonlinear wave equation corresponds to a homoclinic orbit of its traveling wave equation; a kink (or antikink) wave solution corresponds to a heteroclinic orbit (or connecting orbit). Similarly, a periodic orbit of a traveling wave equation corresponds to a periodic travelling wave solution of the nonlinear wave equation. To find all possible bifurcations of solitary waves, and periodic waves, kink and antikink wave of a nonlinear wave equation, we need to investigate the existence of all homoclinic, heteroclinic orbits, and periodic orbits for its traveling wave equation in the parameter space. The solitary wave solution is also called soliton solution. Recently, some new phenomena of soliton are revealed by many works, see [17-26] and the references cited therein.

2.2. Existence of Traveling Wave Solutions for (1). In this subsection, according to the bifurcations of phase portraits in Figures 1 and 2, by using analysis of the phase portraits, we will discuss the existence of smooth solitary traveling wave solutions, kink wave solutions, peakon solutions, compacton solutions, and periodic traveling wave solutions of (1).

Theorem 1. Existence theorem of traveling wave solution. Suppose that $v<0$. Then consider the following.

(i) Equation (1) has infinitely many periodic wave solutions corresponding to a family of close orbits $H(\phi, y)=$ $h$ under the following parametric conditions:

(1) $g_{1}<g<g_{3}, h_{1}<h<h_{2}$, see Figure 1(b);

(2) $g=g_{3}, h_{1}<h<h_{2}$, see Figure 1(c);

(3) $g_{3}<g<g_{2}, h_{3}<h<h_{2}$, see Figure $1(d)$.

(ii) Equation (1) has a solitary wave solution corresponding to the homoclinic orbit $H(\phi, y)=h_{1}$ under the parametric conditions $g_{1}<g<g_{3}$, see Figure $1(b)$. 


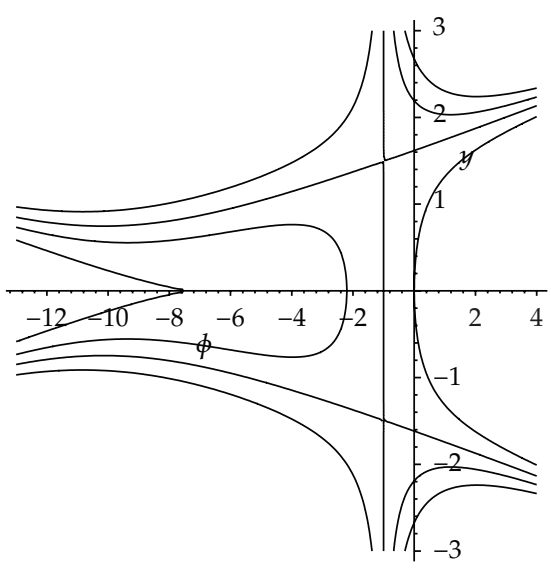

(a) $g=g_{1}$

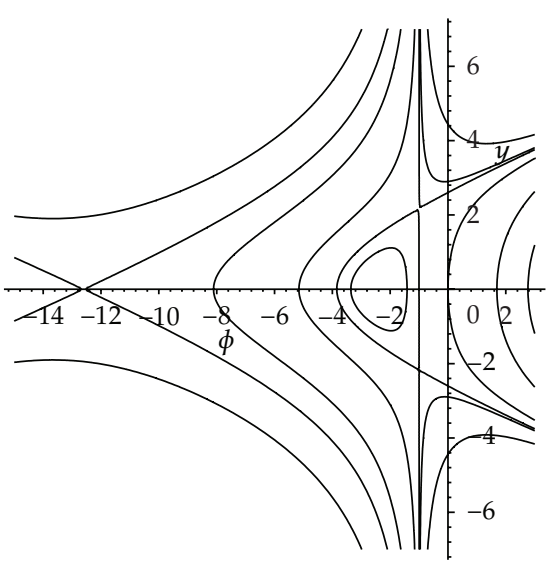

(d) $g_{3}<g<g_{2}$

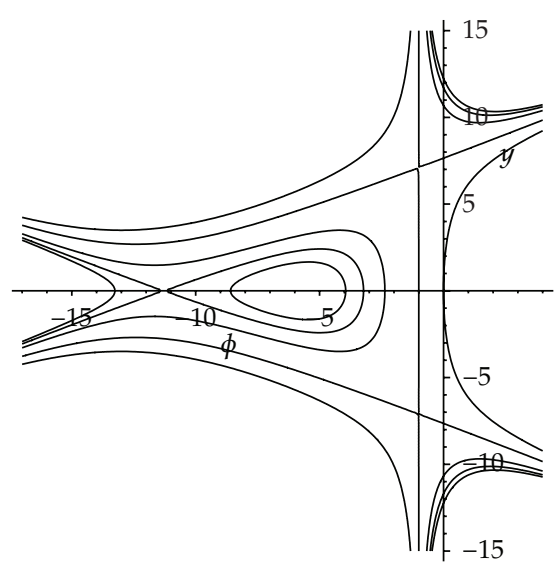

(b) $g_{1}<g<g_{3}$

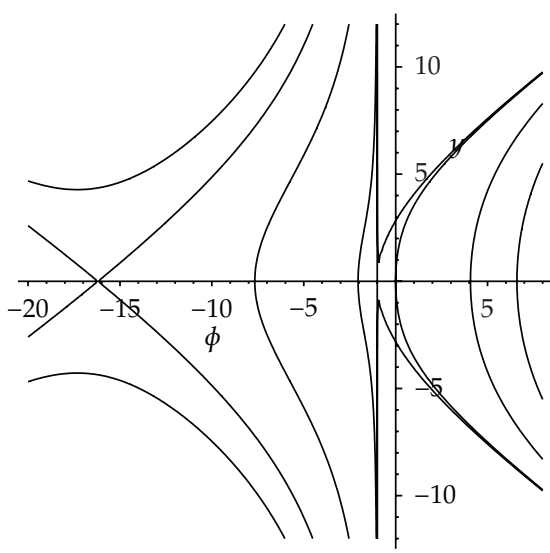

(e) $g=g_{2}$

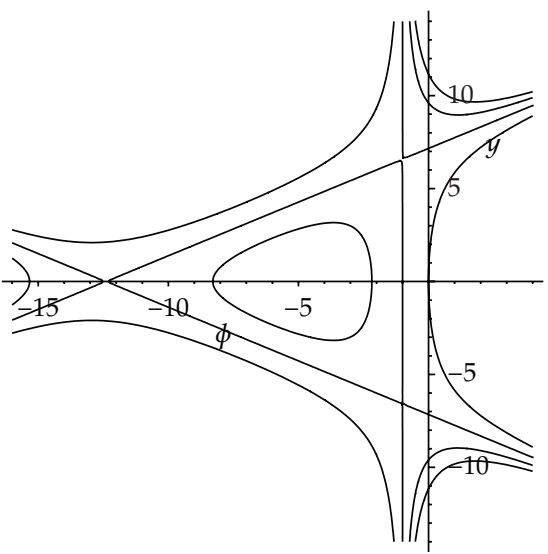

(c) $g=g_{3}$

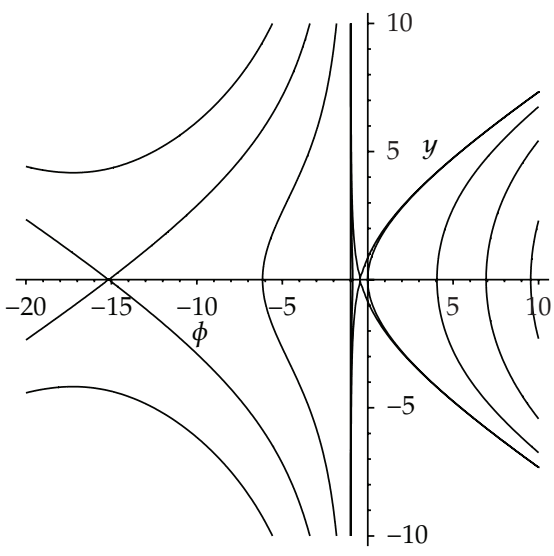

(f) $g>g_{2}$

FIgURE 1: The phase portraits of (7) under different parametric conditions for $v<0$.

(iii) Equation (1) has a solitary cusp wave (peakon) solution corresponding to two heteroclinic orbits $H(\phi, y)=h_{s}$ under the following parametric conditions $g=g_{3}, h_{1}=$ $h_{s}$, see Figure 1(c).

(iv) Equation (1) has a periodic cusp wave solution corresponding to a semilunar orbits $H(\phi, y)=h_{3}$ under the following parametric conditions $g_{3}<g<g_{2}, h_{3}=h_{4}$, see Figure 1(c).

(v) Equation (1) has infinitely many compacton solutions corresponding to a family of bounded open orbits $H(\phi, y)=h$ under the following parametric conditions:

(1) $g_{3}<g<g_{2}, h_{1}<h<h_{3}$, see Figure $1(d)$;

(2) $g=g_{2}, h_{1}<h<h_{3}$, see Figure 1(e);

(3) $g>g_{2}, h_{1}<h<h_{3}$, see Figure $1(f)$.

(vi) Equation (1) has two-half kink wave (generalized kink wave) solutions corresponding to two heteroclinic orbits $H(\phi, y)=h$ under the following parametric conditions:
(1) $g_{3}<g<g_{2}, h=h_{1}$, see Figure $1(d)$;

(2) $g>g_{2}, h=h_{1}$ or $h=h_{2}$, see Figure $1(f)$.

Theorem 2. Existence theorem of traveling wave solution. Suppose that $v>0$. Then consider the following.

(i) Equation (1) has infinitely many periodic wave solutions corresponding to a family of close orbits $H(\phi, y)=$ $h$ under the following parametric conditions:

(1) $c<0, g>g_{2}, h_{1}<h<h_{3}$ and $h_{3}<h<h_{2}$, see Figure 2(a);

(2) $c<0, g=g_{2}, h_{3}<h<h_{2}$, see Figure 2(b);

(3) $c<0, g_{1}<g<g_{2}, h_{1}<h<h_{2}$, see Figure 2(c);

(4) $c>0, g_{1}<g<g_{2}, h_{1}<h<h_{2}$, see Figure $2(f)$;

(5) $c>0, g=g_{2}, h_{1}<h<h_{2}$, see Figure 2(g);

(6) $c>0, g>g_{2}, h_{1}<h<h_{3}$ and $h_{3}<h<h_{2}$, see Figure 2(g).

(ii) Equation (1) has a solitary wave solution corresponding to the homoclinic orbit $H(\phi, y)=h_{1}$ or $H(\phi, y)=h_{2}$ under the parametric conditions: 


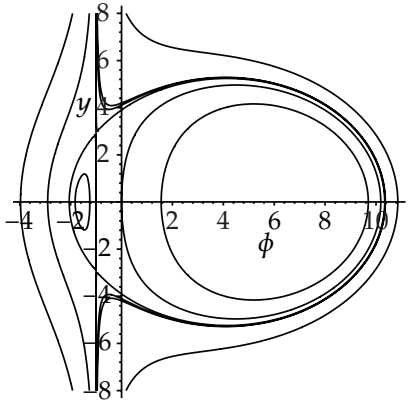

(a) $c<0, g>g_{2}$

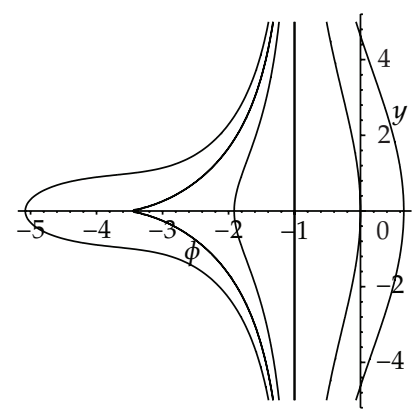

(e) $c>0, g=g_{1}$

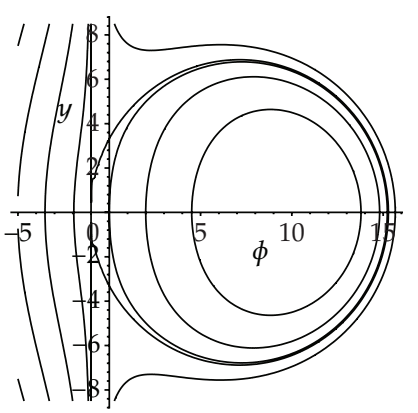

(b) $c<0, g=g_{2}$

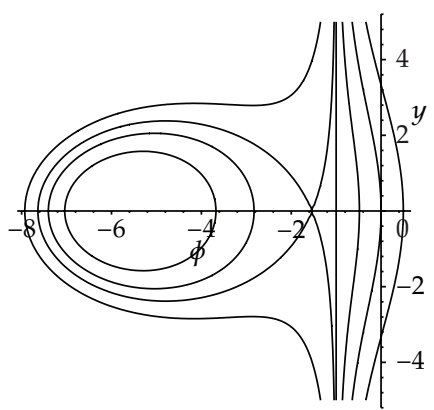

(f) $c>0, g_{1}<g<g_{2}$

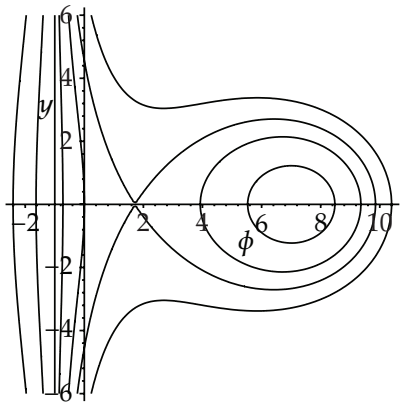

(c) $c<0, g_{1}<g<g_{2}$

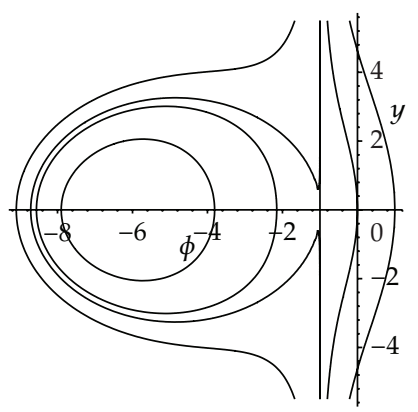

(g) $c>0, g=g_{2}$

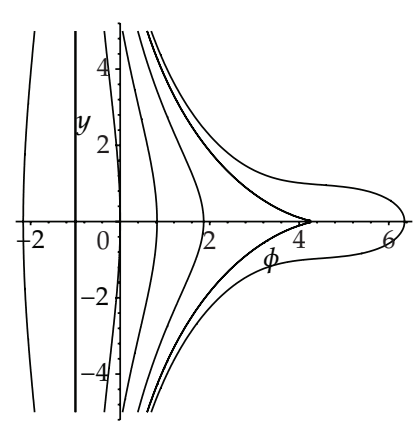

(d) $c<0, g=g_{1}$

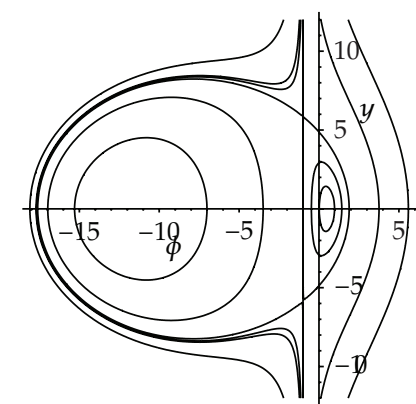

(h) $c>0, g>g_{2}$

FIGURE 2: The phase portraits of (7) under different parametric conditions for $v<0$.

(1) $c<0, g_{1}<g<g_{2}, h=h_{1}$, see Figure 2(c);

(2) $c>0, g_{1}<g<g_{2}, h=h_{2}$, see Figure $2(f)$.

(iii) Equation (1) has a periodic cusp wave solution corresponding to a semilunar orbits $H(\phi, y)=h_{3}$ under the following parametric conditions:

(1) $c<0, g>g_{2}, h_{2}=h_{3}$, see Figure 2(a);

(2) $c>0, g>g_{2}, h_{2}=h_{3}$, see Figure $2(h)$.

(iv) Equation (1) has infinitely many compacton solutions corresponding to a family of bounded open orbits $H(\phi, y)=h$ under the following parametric conditions:

(1) $c<0, g>g_{2}, h>h_{2}$ and $-\infty<h<h_{1}$, see Figure 2(a);

(2) $c<0, g=g_{2}, h>h_{3}$ and $0<h<h_{3}$, see Figure 2(b);

(3) $c<0, g_{1}<g<g_{2}, h>h_{3}$ and $-\infty<h<h_{1}$, see Figure 2(c);

(4) $c<0, g=g_{1}, h>h_{3}$ and $-\infty<h<h_{2}$, see Figure 2(d);

(5) $c>0, g=g_{1}, h_{1}<h<+\infty$ and $-\infty<h<h_{1}$, see Figure 2(e);

(6) $c>0, g_{1}<g<g_{2}, h_{2}<h<+\infty$ and $-\infty<$ $h<h_{1}$, see Figure $2(f)$;

(7) $c>0, g=g_{2}, h_{2}<h<+\infty$ and $h_{1}<h<h_{2}$, see Figure 2(g);

(8) $c>0, g>g_{2}, h_{2}<h<+\infty$ and $-\infty<h<h_{1}$, see Figure $2(g)$. (v) Equation (1) has two-half kink wave (generalized kink wave) solutions corresponding to two heteroclinic orbits $H(\phi, y)=h$ under the following parametric conditions:
(1) $c<0, g=g_{1}, h=h_{1}=h_{2}$, see Figure 2(d);
(2) $c>0, g=g_{1}, h=h_{1}=h_{2}$, see Figure 2(e).

\section{The Exact Travelling Wave Solutions of (1)}

In this section, we discuss exact travelling wave solutions of (1) under some parametric conditions.

Case 1. When $h=h_{s}, g=g_{3}$, system (7) has two heteroclinic orbits, see Figure 1(c); by using (5), their expressions can be written as

$$
y= \pm \sqrt{\frac{1}{-v}}\left(\phi-\frac{3 \beta-3 v-2 \alpha v \lambda}{2 \alpha v}\right) .
$$

Corresponding to these two heteroclinic orbits, (1) has a peakon solution as follows:

$$
u=\lambda+\left(\frac{3(2 c v-v-3 \beta)}{2 \alpha v}\right) e^{-\sqrt{1 /-v}|\xi|}
$$

Case 2. When $h=h_{1}, g_{1}<g<g_{3}, \lambda=(c-1) / \alpha, g=\alpha$, $c=-(\sqrt{2} \alpha v-3 \beta+v) / 2 v$, system (7) has a homoclinic orbit, see Figure 1(b); by using (5), the expression can be written as

$$
y= \pm \sqrt{-\frac{1}{v}}(-2 \sqrt{2}+\phi)(\sqrt{2}+\phi) .
$$


Corresponding to the homoclinic orbit, (1) has a solitary wave solution as follows:

$$
u=\lambda+\frac{\sqrt{2} \tanh ^{2}((1 / 2) \sqrt{-1 / v} \xi)+2 \sqrt{2}}{\operatorname{sech}^{2}((1 / 2) \sqrt{-1 / \nu} \xi)} .
$$

Case 3. When $h=h_{1}, g=g_{2}$, system (7) has two heteroclinic orbits, see Figure 1(e); by using (5), the expression can be written as

$$
y= \pm \sqrt{-\frac{1}{v}}\left(\phi-\phi_{1}\right) \sqrt{\frac{z_{1}-\phi}{\phi_{s}-\phi}} \quad\left(\phi_{1}<\phi<\phi_{s}<z_{1}\right),
$$

where $z_{1}=(5 c v+v-6 \beta-\alpha v \lambda) / \alpha v$. Corresponding to these two heteroclinic orbits, taking $\left(0, \phi_{i}^{0}\right),(i=1,2)$ as the initial values and substituting (14) into the first expression of (4), we have

$$
\int_{\phi_{i}^{0}}^{\phi} \frac{1}{\varphi-\phi_{1}} \sqrt{\frac{\phi_{s}-\varphi}{z_{1}-\varphi}} d \varphi= \pm \int_{0}^{\xi} \sqrt{-\frac{1}{v}} d \widetilde{\xi} .
$$

In order to compute the left integral of (15), we let $\sqrt{\left(\phi_{s}-\varphi\right) /\left(z_{1}-\varphi\right)}=\psi$. First, using the integration by substitution, we compute the indefinite integral $\int(1 /(\varphi-$ $\left.\left.\phi_{1}\right)\right) \sqrt{\left(\phi_{s}-\varphi\right) /\left(z_{1}-\varphi\right)} d \varphi$, see the following computation:

$$
\begin{aligned}
& \int \frac{1}{\varphi-\phi_{1}} \sqrt{\frac{\phi_{s}-\varphi}{z_{1}-\varphi}} d \varphi \\
& =\frac{2\left(\phi_{s}-z_{1}\right)}{z_{1}-\phi_{1}} \int \frac{\psi^{2}}{\left(\psi^{2}-1\right)\left(\psi^{2}-\left(\phi_{s}-\phi_{1}\right) /\left(z_{1}-\phi_{1}\right)\right)} d \psi \\
& =\int\left(\frac{1}{\psi+1}-\frac{1}{\psi+1}\right) d \psi+\delta \int\left(\frac{1}{\psi-\delta}-\frac{1}{\psi+\delta}\right) d \psi \\
& =\ln \left[\frac{\psi+1}{\psi-1}\left(\frac{\psi-\delta}{\psi+\delta}\right)^{\delta}\right] \\
& =\ln \left[\frac{\sqrt{\phi_{s}-\phi}+\sqrt{z_{1}-\phi}}{\sqrt{\phi_{s}-\phi}-\sqrt{z_{1}-\phi}}\left(\frac{\sqrt{\phi_{s}-\phi}-\delta \sqrt{z_{1}-\phi}}{\sqrt{\phi_{s}-\phi}+\delta \sqrt{z_{1}-\phi}}\right)^{\delta}\right],
\end{aligned}
$$

where $\delta=\sqrt{\left(\phi_{s}-z_{1}\right) /\left(z_{1}-\phi_{1}\right)}$. Second, by using (16), completing the integral equation (15), we obtain

$$
\begin{gathered}
\ln \left[\frac{\sqrt{\phi_{s}-\phi}+\sqrt{z_{1}-\phi}}{\sqrt{\phi_{s}-\phi}-\sqrt{z_{1}-\phi}}\left(\frac{\sqrt{\phi_{s}-\phi}-\delta \sqrt{z_{1}-\phi}}{\sqrt{\phi_{s}-\phi}+\delta \sqrt{z_{1}-\phi}}\right)^{\delta}\right] \\
=\ln \Omega_{i} \pm \sqrt{-\frac{1}{v}} \xi
\end{gathered}
$$

where

$$
\begin{array}{r}
\Omega_{i}=\left(\frac{\sqrt{\phi_{s}-\phi_{i}^{0}}+\sqrt{z_{1}-\phi_{i}^{0}}}{\sqrt{\phi_{s}-\phi_{i}^{0}}-\sqrt{z_{1}-\phi_{i}^{0}}}\right)\left(\frac{\sqrt{\phi_{s}-\phi_{i}^{0}}-\delta \sqrt{z_{1}-\phi_{i}^{0}}}{\sqrt{\phi_{s}-\phi_{i}^{0}}+\delta \sqrt{z_{1}-\phi_{i}^{0}}}\right)^{\delta} \\
i=1,2,
\end{array}
$$

with $\phi_{i}^{0} \in\left(\phi_{1}, \phi_{s}\right)$. Finally, substituting $\phi=u-\lambda$ into (18), and then simplifying them, we obtain two generalized kink wave solutions of (1) as follows:

$$
\begin{aligned}
& \left(\frac{\sqrt{\phi_{s}-u+\lambda}+\sqrt{z_{1}-u+\lambda}}{\sqrt{\phi_{s}-u+\lambda}-\sqrt{z_{1}-u+\lambda}}\right)\left(\frac{\sqrt{\phi_{s}-u+\lambda}-\delta \sqrt{z_{1}-u+\lambda}}{\sqrt{\phi_{s}-u+\lambda}+\delta \sqrt{z_{1}-u+\lambda}}\right)^{\delta} \\
& =\Omega_{1} e^{\sqrt{-1 / v} \xi}, \quad \xi \in\left(-\infty, l_{1}\right), \\
& \left(\frac{\sqrt{\phi_{s}-u+\lambda}+\sqrt{z_{1}-u+\lambda}}{\sqrt{\phi_{s}-u+\lambda}-\sqrt{z_{1}-u+\lambda}}\right)\left(\frac{\sqrt{\phi_{s}-u+\lambda}-\delta \sqrt{z_{1}-u+\lambda}}{\sqrt{\phi_{s}-u+\lambda}+\delta \sqrt{z_{1}-u+\lambda}}\right)^{\delta} \\
& =\Omega_{2} e^{-\sqrt{-1 / v} \xi}, \quad \xi \in\left(l_{2},+\infty\right),
\end{aligned}
$$

where $l_{i}=-\sqrt{-v} \ln \Omega_{i},(i=1,2)$.

Case 4. When $g=g_{2}, h=h_{s}$, system (7) has a close orbit, see Figure 2(b); by using (5), the expression can be written as

$$
y= \pm \sqrt{\frac{1}{v}} \sqrt{\left(z_{2}-\phi\right)\left(\phi-\phi_{s}\right)}
$$

where $z_{2}=(6 \beta-3 v-3 c v-\alpha v \lambda) / \alpha v$. Corresponding to this close orbit, (1) has a periodic wave solution as follows:

$$
u=\left(\lambda+\frac{3 v+2 \alpha v \lambda-3 \beta}{2 \alpha v}\right)+\frac{3 v+6 c v-6 \beta}{2 \alpha v} \cos \left(\sqrt{\frac{1}{v}} \xi\right) .
$$

Case 5. When $g=g_{2}, h=0$, system (7) has a open orbit, see Figure 2(g); by using (5), the expression can be written as

$$
\begin{array}{r}
y= \pm \sqrt{\frac{1}{v}} \sqrt{\frac{\left(\phi-z_{3}\right)\left(\phi-z_{4}\right)(0-\phi)}{\phi-\phi_{s}}} \\
\left(z_{3}<z_{4}<\phi_{s}<\phi \leq 0\right),
\end{array}
$$

where $z_{3,4}=(3 v(c-1-\alpha \lambda) \mp \sqrt{\Delta}) / 2 \alpha v, \Delta=3(5 c v+v-6 \beta-$ $\alpha v \lambda)(3 c v+3 v-6 \beta+\alpha v \lambda)$. Corresponding to this open orbit, substituting (23) into the first equation of (4) yields

$$
\int_{\phi_{s}}^{\phi} \sqrt{\frac{\varphi-\phi_{s}}{\left(\varphi-z_{3}\right)\left(\varphi-z_{4}\right)(0-\varphi)}} d \varphi= \pm \int_{0}^{\xi} \sqrt{\frac{1}{v}} d \tilde{\xi} .
$$

By using the formulas 256.02th and 400th in [27], we integrate $\int_{(\phi)_{s}}^{\phi} \sqrt{\left(\varphi-\phi_{s}\right) /\left[\left(\varphi-z_{3}\right)\left(\varphi-z_{4}\right)(0-\varphi)\right]} d \varphi$ of $(24)$, thus we obtain a compacton solution of (1) as follows:

$$
\begin{aligned}
\Pi\left(\operatorname{sn}^{-1}\right. & \left.\left(\sqrt{\frac{z_{4}\left(u-\lambda-\phi_{s}\right)}{\phi_{s}\left(u-\lambda-z_{4}\right)}}, k_{2}\right), \alpha_{2}^{2}\right) \\
& -\operatorname{sn}^{-1}\left(\sqrt{\frac{z_{4}\left(u-\lambda-\phi_{s}\right)}{\phi_{s}\left(u-\lambda-z_{4}\right)}}, k_{2}\right) \\
= & \Omega_{3}\left(\xi_{2}^{*}-|\xi|\right),
\end{aligned}
$$


where $\Pi\left(\varphi, \widetilde{\alpha}^{2}, k\right)$ is the incomplete elliptic integral of the third kind in Legendre's canonical form, and $\alpha_{2}^{2}=\phi_{s} / z_{4}, k_{2}^{2}=$ $\phi_{s}\left(z_{4}-z_{3}\right) /\left[z_{4}\left(\phi_{s}-z_{3}\right)\right], \Omega_{3}=\sqrt{-z_{4}\left(\phi_{s}-z_{3}\right)} /\left(2 \sqrt{v}\left(\phi_{s}-z_{4}\right)\right)$, $\xi_{2}^{*}=\left(\Pi\left(\alpha_{2}^{2}, k_{2}\right)-K\left(k_{2}\right)\right) / \Omega_{3}$.

\section{Conclusion}

In this work, by using the bifurcation theory of dynamic system, the generalization of $\mathrm{KdV}$ equation (1) was studied. According to the analysis of the phase portraits, the existing theorems on solitary wave, cusp wave, periodic wave, periodic cusp wave and compactons are given. In some parametric conditions, exact peakon solution (11), solitary wave solution (13), generalized kink wave solutions (15) and (16), smooth periodic wave solution (19), and compacton solution (21) were obtained. These exact solutions are different from those exact solutions in $[8,12]$.

\section{Acknowledgments}

The authors thank reviewers very much for their useful comments and helpful suggestions. This research is supported by the National Natural Science Foundation of China (11161020) and the Natural Science Foundation of Yunnan Province (2011FZ193).

\section{References}

[1] A. S. Fokas, "On a class of physically important integrable equations," Physica D, vol. 87, no. 1-4, pp. 145-150, 1995.

[2] B. Fuchssteiner and A. S. Fokas, "Symplectic structures, their Bäcklund transformations and hereditary symmetries," Physica $D$, vol. 4, no. 1, pp. 47-66, 1982.

[3] Z. Li and N. R. Sibgatullin, "An improved theory of long waves on the water surface," Journal of Applied Mathematics and Mechanics, vol. 61, pp. 177-482, 1997.

[4] B. Fuchssteiner, "Application of hereditary symmetries to nonlinear evolution equations," Nonlinear Analysis, vol. 3, no. 6, pp. 849-862, 1979.

[5] P. Rosenau and J. M. Hyman, "Compactons: solitons with finite wavelength," Physical Review Letters, vol. 70, no. 5, pp. 564-567, 1993.

[6] J. Li and Z. Liu, "Smooth and non-smooth travelling waves in a nonlinearly dispersive equation," Applied Mathematical Modelling, vol. 25, pp. 41-56, 2000.

[7] Y. Long, B. He, W. Rui, and C. Chen, "Compacton-like and kinklike waves for a higher-order wave equation of Korteweg-de Vries type," International Journal of Computer Mathematics, vol. 83, no. 12, pp. 959-971, 2006.

[8] Q. Bi, "Wave patterns associated with a singular line for a biHamiltonian system," Physics Letters A, vol. 369, no. 5-6, pp. 407-417, 2007.

[9] J. Li and J. Zhang, "Bifurcations of travelling wave solutions for the generalization form of the modified KdV equation," Chaos, Solitons \& Fractals, vol. 21, no. 4, pp. 889-913, 2004.

[10] W. Rui, B. He, Y. Long, and C. Chen, “The integral bifurcation method and its application for solving a family of thirdorder dispersive PDEs," Nonlinear Analysis: Theory, Methods \& Applications, vol. 69, no. 4, pp. 1256-1267, 2008.
[11] R. Weiguo, L. Yao, H. Bin, and L. Zhenyang, "Integral bifurcation method combined with computer for solving a higher order wave equation of KdV type," International Journal of Computer Mathematics, vol. 87, no. 1-3, pp. 119-128, 2010.

[12] W. Rui, C. Chen, X. Yang, and Y. Long, "Some new solitonlike solutions and periodic wave solutions with loop or without loop to a generalized KdV equation," Applied Mathematics and Computation, vol. 217, no. 4, pp. 1666-1677, 2010.

[13] J. Li and Z. Liu, "Traveling wave solutions for a class of nonlinear dispersive equations," Chinese Annals of Mathematics. Series B, vol. 23, no. 3, pp. 397-418, 2002.

[14] H. Junqi, "An algebraic method exactly solving two highdimensional nonlinear evolution equations," Chaos, Solitons \& Fractals, vol. 23, no. 2, pp. 391-398, 2005.

[15] E. Yomba, "The extended F-expansion method and its application for solving the nonlinear wave, CKGZ, GDS, DS and GZ equations," Physics Letters, Section A, vol. 340, no. 1-4, pp. 149160, 2005.

[16] X. Wu, W. Rui, and X. Hong, "Exact traveling wave solutions of explicit type, implicit type, and parametric type for $K(m, n)$ equation," Journal of Applied Mathematics, vol. 2012, Article ID 236875, 23 pages, 2012.

[17] A. Biswas, A. Yildirim, T. Hayat, O. M. Aldossary, and R. Sassaman, "Soliton perturbation theory for the generalized Klein-Gordon equation with full nonlinearity," Proceedings of the Romanian Academy. Series A, vol. 13, no. 1, pp. 32-41, 2012.

[18] H. Triki, A. Yildirim, T. Hayat, O. M. Aldossary, and A. Biswas, "Topological and non-topological soliton solutions of the Bretherton equation," Proceedings of the Romanian Academy. Series A, vol. 13, no. 2, pp. 103-108, 2012.

[19] G. Ebadi, A. H. Kara, M. D. Petkovic, A. Yildirim, and A. Biswas, "Solitons and conserved quantities of the Ito equation," Proceedings of the Romanian Academy. Series A, vol. 133, pp. 215224, 2012.

[20] A. G. Johnpillai, A. Yildirim, and A. Biswas, "Chiral solitons with Bohm potential by Lie group analysis and traveling wave hypothesis," Romanian Journal of Physics, vol. 57, no. 3-4, pp. 545-554, 2012.

[21] H. Triki, S. Crutcher, A. Yildirim, T. Hayat, O. M. Aldossary, and A. Biswas, "Bright and dark solitons of the modified complex Ginzburg Landau equation with parabolic and dual-power law nonlinearity," Romanian Reports in Physics, vol. 64, no. 2, pp. 357-366, 2012.

[22] H. Triki, A. Yildirim, T. Hayat, O. M. Aldossary, and A. Biswas, "Shock wave solution of Benney-Luke equation," Romanian Journal of Physics, vol. 57, no. 7-8, pp. 1029-1034, 2012.

[23] L. Girgis, D. Milovic, S. Konar, A. Yildirim, H. Jafari, and A. Biswas, "Optical Gaussons in birefringent fibers and DWDM systems with intermodal dispersion," Romanian Reports in Physics, vol. 64, no. 3, pp. 663-671, 2012.

[24] H. Triki, A. Yildirim, T. Hayat, O. M. Aldossary, and A. Biswas, "Topological and non-topological solitons of a generalized nonlinear Schrodinger's equation with perturbation terms," Romanian Reports in Physics, vol. 64, no. 3, pp. 672-684, 2012.

[25] S. Crutcher, A. Oseo, A. Yildirim, and A. Biswas, "Oscillatory parabolic law spatial optical solitons," Journal of Optoelectronics and Advanced Materials, vol. 14, no. 1-2, pp. 29-40, 2012.

[26] A. Biswas, K. Khan, A. Rahaman, A. Yildirim, T. Hayat, and O. M. Aldossary, "Bright and dark optical solitons ion birefringent fibers with Hamiltonian perturbations and Kerr law nonlinearity," Journal of Optoelectronics and Advanced Materials, vol. 14, no. 7-8, pp. 571-576, 2012. 
[27] P. F. Byrd and M. D. Friedman, Handbook of Elliptic Integrals for Engineers and Physicists, Die Grundlehren der Mathematischen Wissenschaften in Einzeldarstellungen mit besonderer Berücksichtigung der Anwendungsgebiete, Springer, Berlin, Germany, 1954. 


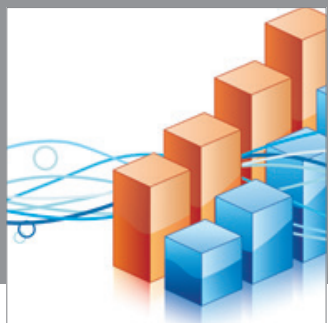

Advances in

Operations Research

mansans

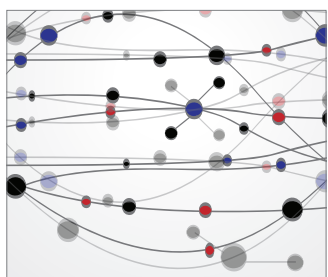

The Scientific World Journal
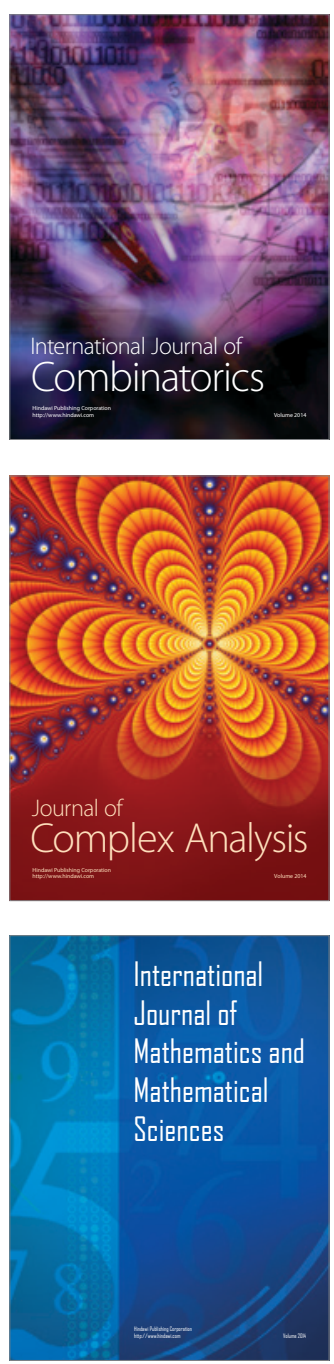
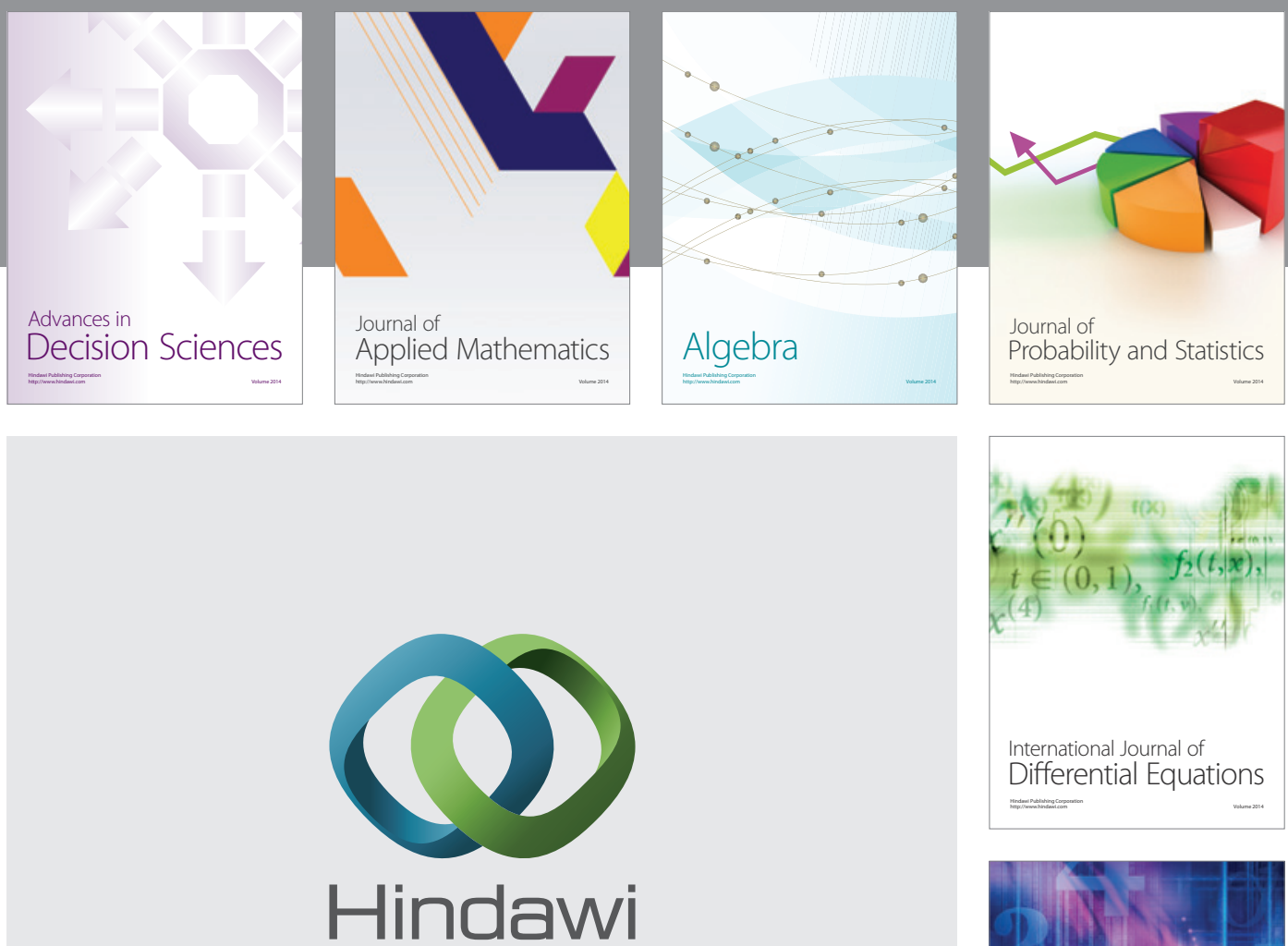

Submit your manuscripts at http://www.hindawi.com
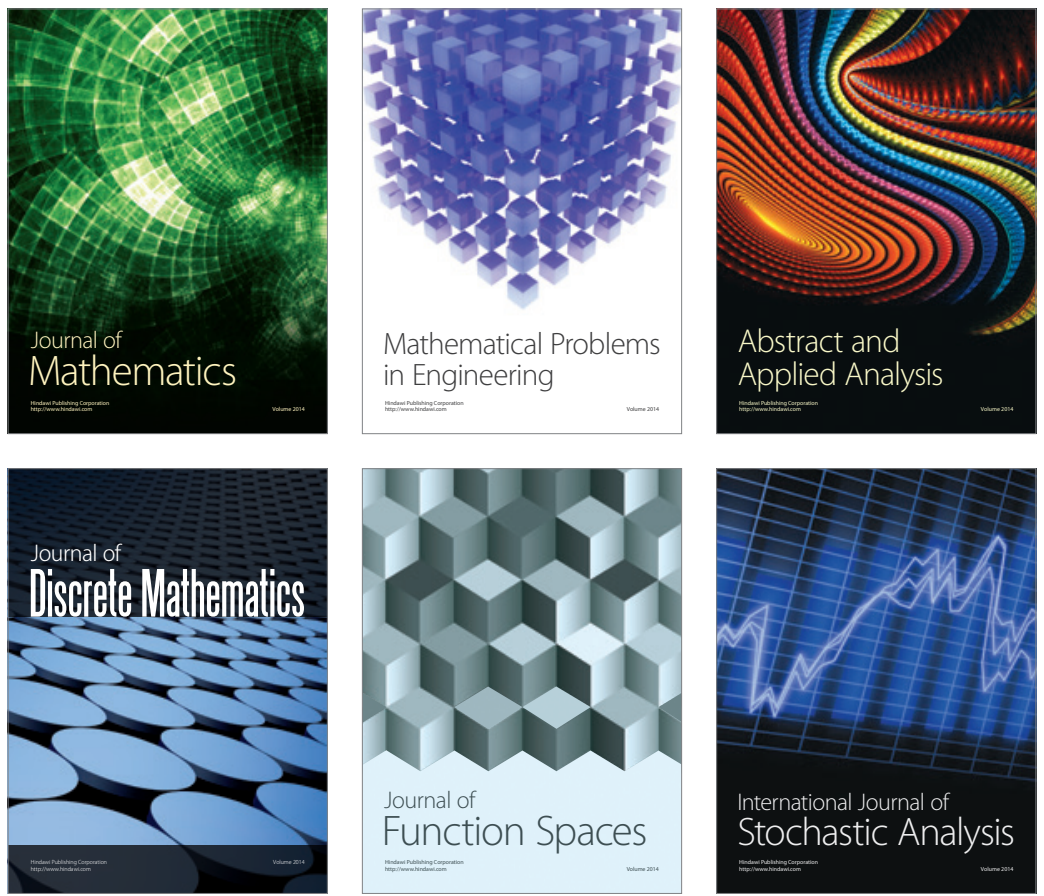

Journal of

Function Spaces

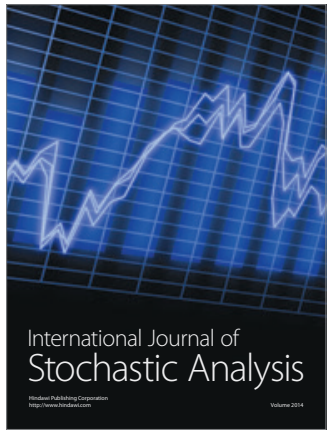

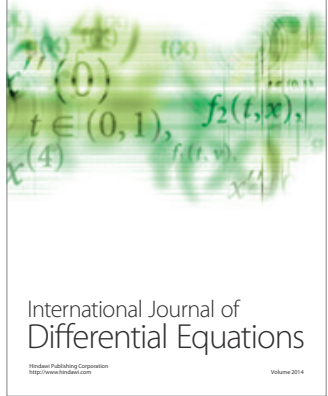
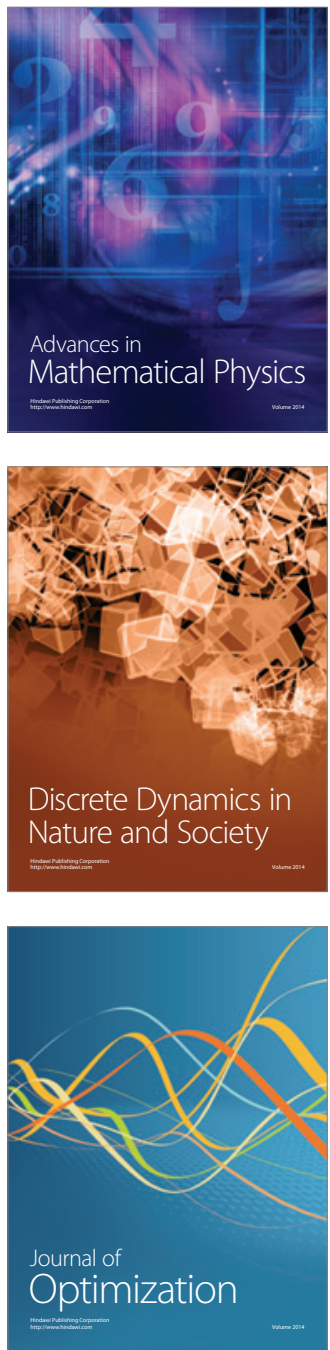